\title{
Data collection in qualitative research
}

\section{David Barrett, ${ }^{1}$ Alison Twycross ${ }^{2}$}

$10.1136 /$ eb-2018-102939

${ }^{1}$ Faculty of Health Sciences, University of Hull, Hull, UK

${ }^{2}$ School of Health and Social Care, London South Bank

University, London, UK

Correspondence to: Dr David Barrett, Faculty of Health Sciences, University of Hull, Hull HU6 7RX, UK; D.I. Barrett@hull.ac.uk
Qualitative research methods allow us to better understand the experiences of patients and carers; they allow us to explore how decisions are made and provide us with a detailed insight into how interventions may alter care. To develop such insights, qualitative research requires data which are holistic, rich and nuanced, allowing themes and findings to emerge through careful analysis. This article provides an overview of the core approaches to data collection in qualitative research, exploring their strengths, weaknesses and challenges.

\section{Interviews}

Collecting data through interviews with participants is a characteristic of many qualitative studies. Interviews give the most direct and straightforward approach to gathering detailed and rich data regarding a particular phenomenon. The type of interview used to collect data can be tailored to the research question, the characteristics of participants and the preferred approach of the researcher. Interviews are most often carried out faceto-face, though the use of telephone interviews to overcome geographical barriers to participant recruitment is becoming more prevalent.

The key variation between interview types relates to the degree of structure. An open or unstructured interview will often be based on a single question, with the interviewer and interviewee then shaping the conversation in real time, rather than following a prewritten schedule. This can be particularly suited to methods in which participants are being encouraged to tell a story of their life or experiences, such as narrative enquiry. An example of the type of study in which these open, conversational interviews are well suited was an exploration of the impact of time on the work of Registered Nurses. $^{2}$

A common approach in qualitative research is the semistructured interview, where core elements of the phenomenon being studied are explicitly asked about by the interviewer. A well-designed semistructured interview should ensure data are captured in key areas while still allowing flexibility for participants to bring their own personality and perspective to the discussion. Finally, interviews can be much more rigidly structured to provide greater control for the researcher, essentially becoming questionnaires where responses are verbal rather than written.

Deciding where to place an interview design on this 'structural spectrum' will depend on the question to be answered and the skills of the researcher. A very structured approach is easy to administer and analyse but may not allow the participant to express themselves fully. At the other end of the spectrum, an open approach allows for freedom and flexibility, but requires the researcher to walk an investigative tightrope that maintains the focus of an interview without forcing participants into particular areas of discussion.
An example of a semistructured interview schedule for a study exploring surgeons' perceptions of postoperative pain $^{3}$ can be seen in box 1 . An interview schedule such as this is normally developed prior to the start of the study following an extensive review of the literature and often also draws on the researcher's clinical experience. Before data collection commences, the interview schedule is often reviewed by a small group of subject experts to ensure all key issues have been included.

Interviews present several challenges to researchers. Most interviews are recorded and will need transcribing before analysing. This can be extremely time-consuming, with 1 hour of interview requiring 5-6hours to transcribe. ${ }^{4}$ The analysis itself is also time-consuming, requiring transcriptions to be pored over word-for-word and line-by-line. Interviews also present the problem of bias the researcher needs to take care to avoid leading questions or providing non-verbal signals that might influence the responses of participants.

\section{Focus groups}

The focus group is a method of data collection in which a moderator/facilitator (usually a coresearcher) speaks with a group of 6-12 participants about issues related to the research question. As an approach, the focus group offers qualitative researchers an efficient method of gathering the views of many participants at one time. Also, the fact that many people are discussing the same issue together can result in an enhanced level of debate, with the moderator often able to step back and let the focus group enter into a free-flowing discussion. ${ }^{5}$ This provides an opportunity to gather rich data from a specific population about a particular area of interest, such as barriers perceived by student nurses when trying to communicate with patients with cancer. ${ }^{6}$

From a participant perspective, the focus group may provide a more relaxing environment than a one-to-one interview; they will not need to be involved with every part of the discussion and may feel more comfortable expressing views when they are shared by others in the group. Focus groups also allow participants to 'bounce' ideas off each other which sometimes results in different perspectives emerging from the discussion. However, focus groups are not without their difficulties. As with interviews, focus groups provide a vast amount of data to be transcribed and analysed, with discussions often lasting 1-2 hours. Moderators also need to be highly skilled to ensure that the discussion can flow while remaining focused and that all participants are encouraged to speak, while ensuring that no individuals dominate the discussion. ${ }^{7}$

\section{Observation}

Participant and non-participant observation are powerful tools for collecting qualitative data, as they give nurse researchers an opportunity to capture a wide 


\section{Box 1 Example of an interview schedule ${ }^{3}$}

- What do you think is the most effective way of assessing a child's pain?

- Have you come across any issues that make it difficult to assess a child's pain?

- What pain-relieving interventions do you find most useful and why?

- When managing pain in children what is your overall aim?

- Whose responsibility is pain management?

- What involvement do you think parents should have in their child's pain management?

- What involvement do children have in their pain management?

- Is there anything that currently stops you managing pain as well as you would like?

-What would help you manage pain better?

array of information-such as verbal and non-verbal communication, actions (eg, techniques of providing care) and environmental factors-within a care setting. Another advantage of observation is that the researcher gains a first-hand picture of what actually happens in clinical practice. ${ }^{8}$ If the researcher is adopting a qualitative approach to observation they will normally record field notes. Field notes can take many forms, such as a chronological log of what is happening in the setting, a description of what has been observed, a record of conversations with participants or an expanded account of impressions from the fieldwork. ${ }^{910}$

As with other qualitative data collection techniques, observation provides an enormous amount of data to be captured and analysed-one approach to helping with collection and analysis is to digitally record observations to allow for repeated viewing. ${ }^{11}$ Observation also provides the researcher with some unique methodological and ethical challenges. Methodologically, the act of being observed may change the behaviour of the participant (often referred to as the 'Hawthorne effect'), impacting on the value of findings. However, most researchers report a process of habitation taking place where, after a relatively short period of time, those being observed revert to their normal behaviour. Ethically, the researcher will need to consider when and how they should intervene if they view poor practice that could put patients at risk.

\section{Conclusion}

The three core approaches to data collection in qualitative research-interviews, focus groups and observation-provide researchers with rich and deep insights. All methods require skill on the part of the researcher, and all produce a large amount of raw data. However, with careful and systematic analysis ${ }^{12}$ the data yielded with these methods will allow researchers to develop a detailed understanding of patient experiences and the work of nurses.

\section{Competing interests None declared.}

Patient consent Not required.

Provenance and peer review Commissioned; internally peer reviewed.

- Article author(s) (or their employer(s) unless otherwise stated in the text of the article) 2018. All rights reserved. No commercial use is permitted unless otherwise expressly granted.

\section{References}

1 Smith EM. Telephone interviewing in healthcare research: a summary of the evidence. Nurse Res 2005;12:32-41.

2 Chan EA, Jones A, Wong K. The relationships between communication, care and time are intertwined: a narrative inquiry exploring the impact of time on registered nurses' work. J Adv Nurs 2013;69:2020-9.

3 Twycross AM, Williams AM, Finley GA. Surgeons' aims and pain assessment strategies when managing paediatric post-operative pain: A qualitative study. J Child Health Care 2015;19:513-23.

4 Bryman A. Social research methods. 4th edn. Oxford University Press: Oxford, 2012.

5 Plummer P. Focus group methodology. Part 1: Design considerations. Int J Ther Rehabil 2017;24:297-301.

6 Lin MF, Hsu WS, Huang MC, et al. "I couldn't even talk to the patient": barriers to communicating with cancer patients as perceived by nursing students. Eur J Cancer Care 2017;26:e12648.

7 Onwuegbuzie AJ, Dickinson WB, Leech NL, et al. A qualitative framework for collecting and analyzing data in focus group research. Int J Qual Methods 2009;8:1-21.

8 Twycross A, Shorten A. Using observational research to obtain a picture of nursing practice. Evid Based Nurs 2016;19:66-7.

9 Emerson RM, Fretz RI, Shaw LL. Writing ethnographic fieldnotes. 2nd edn. Chicago: University of Chicago Press, 2001.

10 Spradley J. Participant observation. Fort Worth, Texas: Harcourt Brace, 1980.

11 Meriläinen M, Kyngäs H, Ala-Kokko T. 24-hour intensive care: an observational study of an environment and events. Intensive Crit Care Nurs 2010;26:246-53.

12 Seers K. Qualitative data analysis. Evid Based Nurs 2012;15:2. 